\title{
Cholestatic Jaundice Induced by Duloxetine in a Patient with Major Depressive Disorder
}

\author{
Young-Min Park ${ }^{\circledR}$, Bun-Hee Lee ${ }^{2}$, Heon-Jeong Lee ${ }^{3}$ and Seung-Gul Kang ${ }^{4}$ \\ ${ }^{1}$ Department of Neuropsychiatry, Ilsan Paik Hospital, Inje University College of Medicine, Goyang, Korea \\ ${ }^{2}$ KARF Hospital, Goyang, Korea \\ ${ }^{3}$ Department of Psychiatry, Anam Hosipital, Korea University College of Medicine, Seoul, Korea \\ ${ }^{4}$ Saessac Hospital, Uijeongbu, Korea
}

Duloxetine is a balanced and potent serotonin and noradrenaline reuptake inhibitor (SNRI) and has adverse effects that are commonly associated with such drugs, including nausea, dry mouth, constipation, insomnia, and dizziness. Recently, duloxetine-induced liver injury has also been observed in patients with preexisting liver disease or chronic alcohol use. We investigated the effects of duloxetine in a healthy young adult with major depressive disorder (MDD) but no risk factors, and found that his total bilirubin level increased to $3.3 \mathrm{mg} / \mathrm{dL}$ and he developed jaundice after 5 months of duloxetine treatment. Discontinuation of duloxetine treatment saw his total bilirubin level decrease to $1.8 \mathrm{mg} / \mathrm{dL}$. Thus, the administration of duloxetine might induce liver injury in a patient with MDD. However, the limitations of this single case report must be acknowledged. Although the cause of hepatic dysfunction in this case remains to be elucidated, clinicians should monitor liver function carefully after duloxetine treatment. Further investigations with a larger sample are needed.

Psychiatry Investig 2010;7:228-230

Key Words Duloxetine, Liver injury, Hepatic dysfunction, Hepatotoxicity, Major depressive disorder.

\section{INTRODUCTION}

Duloxetine, one of a series of serotonin and noradrenaline reuptake inhibitors (SNRIs), was released in Korea in 2009 as a treatment for major depressive disorder (MDD), generalized anxiety disorder (GAD), and diabetic peripheral neuropathic pain (DPNP). Duloxetine is a balanced and potent SNRI, and has adverse effects that are common to the other SNRIs, including nausea, dry mouth, constipation, insomnia, and dizziness. ${ }^{1}$ Duloxetine-induced liver injury has also recently been observed in patients with preexisting liver disease. ${ }^{2}$ During the first 2 years after the release of duloxetine, 406 cases of events potentially relating to hepatic dysfunction were reported. ${ }^{3}$ Thus, the information sheet provided with duloxetine is now required state that it should not be used in patients with any form

Received: May 12, 2010 Revised: June 30, 2010

Accepted: August 11, 2010 Available online: August 30, 2010

$\triangle$ Correspondence: Young-Min Park, MD, PhD

Department of Neuropsychiatry, Ilsan Paik Hospital, Inje University College of Medicine, 2240 Daehwa-dong, Ilsanseo-gu, Goyang 411-706, Korea

Tel: +82-31-910-7260, Fax: +82-31-910-7268

E-mail: medipark@hanmail.net

(a) This is an Open Access article distributed under the terms of the Creative Commons Attribution Non-Commercial License (http://creativecommons.org/licenses/bync/3.0) which permits unrestricted non-commercial use, distribution, and reproduction in any medium, provided the original work is properly cited. of chronic liver disease or in those with chronic alcohol use. ${ }^{2}$

We describe herein a case of jaundice in a patient with MDD who was medicated with duloxetine but had no risk factors. A MEDLINE search has revealed no other report related to hyperbilirubinemia alone induced by duloxetine, and thus this appears to be the first reported case thereof.

\section{CASE}

A 22-year-old Korean male visited our clinic in 2009 due to the onset of a major depressive episode. The presenting symptoms were depressed mood, avolition, idea of reference, insomnia, guilty feeling, and suicidal ideation. The last symptom resulted in him being admitted to a closed psychiatric ward.

The patient had no medical history and no history of substance abuse; his laboratory tests were also normal. He was diagnosed with MDD. He was initially treated with mirtazapine at $15 \mathrm{mg}$, which was subsequently escalated to $45 \mathrm{mg}$. However, the patient had only a partial response to this drug. As a result, an initial dose of $30 \mathrm{mg}$ duloxetine was added to mirtazapine, and subsequently escalated to $60 \mathrm{mg}$. The patient's symptomatology largely remitted after this combination treatment, and so he was discharged to day-clinic treatment. Just prior to 
his discharge, laboratory tests were repeated, and all of them (including the liver function test) produced normal results; his total bilirubin was $1.0 \mathrm{mg} / \mathrm{dL}$ and alkaline phosphatase (ALP) was $88 \mathrm{U} / \mathrm{L}$.

After discharge, the patient maintained the mirtazapine (45 $\mathrm{mg}$ ) and duloxetine $(60 \mathrm{mg})$ combination therapy for 3 months. During this time he developed jaundice in his eyes. Liver function tests were repeated again, and although he did not complain of any symptoms, his total bilirubin level had increased to $3.3 \mathrm{mg} / \mathrm{dL}$, while other test results were within normal limits (e.g., ALP 111 U/L). Duloxetine was discontinued immediately. At 1 month after discontinuation of duloxetine, the patient's total bilirubin had decreased to $1.8 \mathrm{mg} / \mathrm{dL}$, ALP remained normal at $109 \mathrm{U} / \mathrm{L}$, and the jaundice had disappeared.

\section{DISCUSSION}

Drug-induced liver injury (DILI) is one of the most common reasons for drug discontinuation, ${ }^{4}$ and is the most common cause of acute liver failure. ${ }^{5}$ DILI is most often mediated by an idiosyncratic process that has been divided into two parts: metabolic and immune-mediated. ${ }^{6,7}$ The pathology of DILI is divided into hepatocellular, cholestatic, and mixed hepatocellular-cholestatic injury, depending on the particular abnormality detected in the liver function test.-8 The US Food and Drug Administration (FDA) defines DILI as a persistent elevation of at least three times the upper limit of normal (ULN) in alanine transaminase (ALT) levels accompanied by jaundice (total bilirubin greater than or equal to twice the ULN). ${ }^{7}$

Duloxetine was approved by the US FDA in 2004. However, in late 2005, a "Dear Health Care Professional" letter was sent by Eli Lilly and Company. In this letter, the company mentioned that there had been some postmarketing reports of cholestatic jaundice and hepatitis in patients with chronic liver disease or cirrhosis receiving duloxetine. Thus, a note was added to the product labeling to the effect that duloxetine should not ordinarily be prescribed to patients with chronic alcohol use or where there was evidence of chronic liver disease. ${ }^{2}$ Thus, individuals with preexisting chronic liver disease or those consuming significant amounts of alcohol may be at a greater risk of duloxetine-induced liver injury. ${ }^{8}$

The hepatic dysfunction caused by duloxetine was first observed during preclinical studies and in human clinical trials. Preclinical rodent and canine studies showed hepatic dysfunction consistent with microsomal enzyme induction, but no overt liver injury. ${ }^{9}$ However, some clinical studies did find an association between liver injury and medication with duloxetine. It was reported that duloxetine treatment for DPNP was associated with a significant increase in ALP levels..$^{10}$ In particular, the high-dose group (duloxetine $60 \mathrm{mg}$ twice daily) ex- hibited a significant increase in $\gamma$-glutamyl transferase. It was also reported that duloxetine was associated with an elevation in aminotransferases to more than three times the ULN in 1\% of patients. In patients with a normal baseline ALT, both ALT and aspartate transaminase values peaked at 8 weeks, ALP steadily increased to a maximum value at 52 weeks, and mean total bilirubin levels remained unchanged. ${ }^{11}$

In the one published case related to duloxetine-induced liver injury, hepatic failure occurred 6 weeks after dose escalation from 30 to $60 \mathrm{mg}$ daily. ${ }^{12}$ Like our patient, that patient had received combination therapy of duloxetine and mirtazapine. Thus, it is unclear whether both agents were responsible for the hepatic failure or duloxetine alone. However, it has been pointed out that liver injury associated with mirtazapine is rare. ${ }^{13}$ The situation in our case is clearer than in the previous one, because the discontinuation of duloxetine improved the jaundice and decreased the total bilirubin levels, in spite of persisting mirtazapine treatment. Thus, it is possible that duloxetine alone is responsible for hepatic dysfunction.

Our patient showed a unique pattern of duloxetine-induced hepatic dysfunction. First, he was a healthy young adult with no risk factors, such as preexisting liver disease or chronic alcohol use. Second, the total bilirubin level of our patient was $3.3 \mathrm{mg} / \mathrm{dL}$, while the results of other liver function tests were within normal limits. In particular, ALP related to cholestatic injury was also within the normal range, although it did increase slightly from 88 to $111 \mathrm{U} / \mathrm{L}$. However, it should be noted that ALP steadily increased to a maximum value at week 52 after duloxetine initiation. ${ }^{11}$

It may be concluded that the administration of duloxetine can induce liver injury in a patient with MDD. However, the limitations of this single case report must be acknowledged. Although the mechanism underlying the observed hepatic dysfunction in this case remains unclear, clinicians should monitor liver function carefully after duloxetine treatment. Further investigations with a larger sample are needed.

\section{REFERENCES}

1. Hudson JI, Wohlreich MM, Kajdasz DK, Mallinckrodt CH, Watkin JG, Martynov OV. Safety and tolerability of duloxetine in the treatment of major depressivedisorder: analysis of pooled data from eight placebo-controlled clinical trials. Hum Psychopharmacol 2005;20:327-341.

2. CYMBALTA (duloxetine hydrochloride) delayed-release capsules. Product labeling. Indianapolis, IN: Eli Lilly and Company; 2005.

3. Wernicke J, Acharya N, Strombom I, Gahimer JL, D'Souza DN, DiPietro N, et al. Hepatic effects of duloxetine-II: spontaneous reports and epidemiology of hepatic events. Curr Drug Saf 2008;3:143-153.

4. Björnsson E. Drug-induced liver injury: Hy's rule revisited. Clin Pharmacol Ther 2006;79:521-528.

5. Ostapowicz G, Fontana RJ, Schiødt FV, Larson A, Davern TJ, Han SH, et al. Results of a prospective study of acute liver failure at 17 tertiary care centers in the United States. Ann Intern Med 2002;137:947-954.

6. Bleibel W, Kim S, D'Silva K, Lemmer ER. Drug-induced liver injury: 
review article. Dig Dis Sci 2007;52:2463-2471.

7. Holt MP, Ju C. Mechanisms of drug-induced liver injury. AAPS J 2006; 8:E48-E54.

8. McIntyre RS, Panjwani ZD, Nguyen HT, Woldeyohannes HO, Alsuwaidan M, Soczynska JK, et al. The hepatic safety profile of duloxetine: a review. Expert Opin Drug Metab Toxicol 2008;4:281-285.

9. Wohlreich MM, Acharya N, Strombom I, Kuritzky L, Robinson M, Heinloth AN, et al. Answers to the most common questions about the hepatic safety profile of duloxetine. Postgrad Med 2008;120:111-118.

10. Raskin J, Wang F, Pritchett YL, Goldstein DJ. Duloxetine for patients with diabetic peripheral neuropathic pain: a 6-month open-label safety study. Pain Med 2006;7:373-385.

11. Wernicke J, Pangallo B, Wang F, Murray I, Henck JW, Knadler MP, et al. Hepatic effects of duloxetine-I: non-clinical and clinical trial data. Curr Drug Saf 2008;3:132-142.

12. Hanje AJ, Pell LJ, Votolato NA, Frankel WL, Kirkpatrick RB. Case report: fulminant hepatic failure involving duloxetine hydrochloride. Clin Gastroenterol Hepatol 2006;4:912-917.

13. Lucena MI, Carvajal A, Andrade RJ, Velasco A. Antidepressant-induced hepatotoxicity. Expert Opin Drug Saf 2003;2:249-262. 